\title{
En acción: para mejorar el acceso a la atención óptima para todos los pacientes con inmunodeficiencias primarias*
}

\section{Into action: Improving access \\ to optimum care for all primary immunodeficiency patients}

Francisco J. Espinosa-Rosales, ${ }^{1}$ Antonio Condino-Neto, ${ }^{2}$ José L. Franco, ${ }^{3}$ Ricardo U. Sorensen ${ }^{4,5}$

Las inmunodeficiencias primarias (IDP) constituyen un grupo de más de 300 defectos innatos del sistema inmunitario, tanto en sus componentes hematopoyéticos como en los no hematopoyéticos. Dichos defectos pueden presentarse con un amplio espectro de manifestaciones clínicas, ya sea con infecciones (comunes recurrentes, comunes graves, o raras y graves), inflamación, autoinmunidad, malignidad, o alergia. Anteriormente clasificadas como "enfermedades raras", las inmunodeficiencias primarias no son tan raras como se pensaba: actualmente se estima que éstas afectan a más de seis millones de personas en el mundo, sin distinciones geográficas, de sexo o edad.

Debido a que las inmunodeficiencias primarias pueden presentarse bajo la forma de infecciones "comunes", la falta de conocimiento y familiaridad ha derivado en que de 70 a $90 \%$ de las personas afectadas por estas inmunodeficiencias no sean diagnosticadas, toda vez que los profesionales de la salud tratan dichas infecciones asociadas con inmunodeficiencias primarias como cualquiera otra "infección común". En respuesta a la necesidad urgente de despertar conciencia acerca de este padecimiento, organizaciones como la Sociedad Africana para las Inmunodeficiencias (ASID), Sociedad Europea para Inmunodeficiencias (ESID), la Sociedad Latinoamericana para Inmunodeficiencias (LASID), la Sociedad de Inmunología Clínica (CIS); y fundaciones dedicadas a las inmunodeficiencias

\footnotetext{
${ }^{1}$ Unidad de Inmunología y Alergia, Instituto Nacional de Pediatría, Ciudad de México, México

${ }^{2}$ Departamento de Inmunología, Instituto de Ciencias Biomédicas, Universidad de Sao Paulo, Sao Paulo, SP, Brasil

${ }^{3}$ Grupo de Inmunodeficiencias Primarias, Escuela de Medicina, Universidad de Antioquia, Medellín, Colombia

${ }^{4}$ Departamento de Pediatría, Louisiana State University Health Science Center, New Orleans, La

${ }^{5}$ Facultad de Medicina, Universidad de La Frontera, Temuco, Chile
}

*Publicado originalmente en inglés: Francisco J. Espinosa-Rosales FJ, Condino-Neto A, José L. Franco JL, Sorense RU. Into action: Improving access to optimum care for all primary immunodeficiency patients. J Clin Immunol [on line]. 2016;1-3e. Modififcado por Revista Alergia México como apoyo a la difusión de la Semana Mundial de Inmunodeficiencias Primarias, 22-29 de abril de 2016

Recibido: 18 de marzo 2016

Aceptado: 1 de abril 2016

Correspondencia

Dr. Francisco J. Espinosa-Rosales

Publicaciones Médicas, Instituto Nacional de

Pediatría, Av. Insurgentes Sur 3700-C, Col. Insurgentes Cuicuilco, Del. Coyoacán, 04530 Ciudad de México, México.

Tel/fax: 52 (55) 1084 0000, extensión 1112.

espinosa_francisco@yahoo.com.mx

Este artículo debe citarse como Espinosa-Rosales FJ, Condino-Neto A, Franco JL, Sorensen RU. En acción: para mejorar el acceso a la atención óptima para todos los pacientes con inmunodeficiencias primarias. Rev Alerg Méx. 2016 abr-jun;63(2):109-112. 
primarias, como la Fundación de Deficiencia Inmune (IDF), y la Fundación Jeffrey Modell (JMF), así como organizaciones de pacientes como la Organización de Pacientes para Inmunodeficiencias Primarias (IPOPI) han implementado y apoyado, a lo largo de más de dos décadas, campañas y actividades en todo el mundo, dirigidas a médicos y al público en general para incrementar el diagnóstico temprano y el tratamiento apropiado y continuado de las inmunodeficiencias primarias, con miras a reducir la morbilidad y mortalidad asociadas con estos defectos.

Desde el 2011 una campaña global anual para incrementar el conocimiento y familiaridad (la Semana Mundial de las Inmunodeficiencias Primarias) se inició bajo el Ilamado a la acción: "Evalúa, Diagnostica, Trata", y se celebra cada abril con el apoyo de una red global de partes interesadas en las inmunodeficiencias primarias (www.worldpiweek.org), para acercar a todos los grupos activos en el campo alrededor de una plataforma común, y así fortalecer el impacto de sus actividades a nivel local.

Como resultado de tales actividades, y gracias a un número creciente de tratamientos capaces de salvar vidas que han sido desarrollados a lo largo de los últimos 60 años, el número de pacientes con inmunodeficiencias primarias diagnosticados y tratados sigue creciendo cada año.

Sin embargo, a pesar de estos progresos, resulta profundamente preocupante que el acceso al tratamiento tenga variaciones importantes en los distintos continentes e, incluso, de manera significativa en países de una misma región. Por ejemplo, el pronto acceso a un trasplante de células madre y a terapia génica para varios tipos de inmunodeficiencias primarias es la regla en la mayor parte de países de Norteamérica y Europa, mientras que está disponible en apenas un número muy pequeño de centros en Latinoamérica y África. ¿Es esto aceptable? De manera similar, mientras que en la mayor parte de países europeos los sistemas de salud nacionales proporcionan o reembolsan el tratamiento con inmunoglobulina G ( $\operatorname{lgG}$ ) intravenosa o subcutánea, estos tratamientos no siempre son cubiertos en los países más pobres, aun cuando existe evidencia abundante de que el diagnóstico temprano y el tratamiento apropiado de pacientes con inmunodeficiencias primarias salvan vidas, mejoran la calidad de vida de los pacientes y ahorran costos, además de prevenir morbilidad innecesaria.

En vista de tales discrepancias, este año las partes interesadas, unidas bajo el estandarte de la Semana Mundial de las Inmunodeficiencias Primarias, levantan sus voces una vez más, para convocar a tomadores de decisiones, autoridades de salud, financiadores de sistemas de atención, y profesionales de la salud, a que se pongan en marcha los mecanismos relevantes que garanticen que todos los pacientes con inmunodeficiencias primarias puedan recibir tratamientos apropiados, seguros y eficientes; así como un cuidado óptimo, tanto por su propio beneficio, como para beneficio a largo plazo del sistema de salud.

Los pacientes con inmunodeficiencias primarias requieren acceso expedito a antibióticos antifúngicos y antibacterianos profilácticos, y un acceso continuo al tratamiento de reemplazo con inmunoglobulina. Además, algunos pacientes requieren trasplante de células madre hematopoyéticas $(\mathrm{TCH})$ o terapia génica, así como medicina de emergencia de acuerdo con sus necesidades y conforme a las recomendaciones de sus especialistas.

Como tratamiento incluido en la lista de la OMS de medicamentos esenciales para niños y adultos, ${ }^{1}$ y al ser un tratamiento efectivo que salva vidas en la mayoría de los pacientes con inmunodeficiencias primarias, la IgG debería facilitarse a 
todos los pacientes con estas inmunodeficiencia en el mundo. Más específicamente, dado que no hay un solo producto o método de administración de IgG que sea apropiado para todos los pacientes con inmunodeficiencias primarias, todos los países y centros de inmunodeficiencias primarias deberían tener acceso a una gama amplia de productos de IgG para poder proporcionar un tratamiento óptimo a cada paciente. También deberían implementarse mecanismos de financiamiento que garanticen la disponibilidad y la dosificación óptima de la lgG, y que de esa manera se mejore la calidad de vida de los pacientes y se evite un mayor daño orgánico, al mismo tiempo que se reducen los costos de tratamiento.

Más aún, todos los pacientes que lo necesiten deberían recibir trasplante de células madre hematopoyéticas o TG sin importar donde vivan. Se ha demostrado que el trasplante de células madre hematopoyéticas temprano cura varias de las formas más graves de inmunodeficiencias primarias. El tamizaje neonatal sistemático para inmunodeficiencia combinada grave y linfopenia severa de células $T$ con el método de cuantificación de círculos de escisión de células T está disponible en muy pocos países del mundo, pero ha mostrado ser capaz de reducir de manera muy significativa el costo de un trasplante de células madre hematopoyéticas. ${ }^{2}$

Desafortunadamente, a causa de la falta de infraestructura o de personal calificado, el acceso a estos procedimientos es muy difícil en escenarios de menos recursos, y el tiempo de espera es con frecuencia tan largo que muchos pacientes sufren complicaciones graves debidas a infecciones recurrentes. La falta de un donador compatible es también un problema común. Los registros de donadores de médula ósea deberían ser promovidos en cada país para incrementar las probabilidades de realizar un trasplante de células madre hematopoyéticas con la mejor compatibilidad y, por lo tanto, el mejor resultado.
Existen varias formas de inmunodeficiencias primarias letales y graves, en las que la TG ha demostrado ser efectiva y segura, por lo que se está convirtiendo en el procedimiento estándar, especialmente en casos en los que no hay disponible un donador idéntico. ${ }^{3}$ Infortunadamente, en la actualidad se realiza solo en muy pocos centros de alta especialización, en países de ingresos elevados. Las autoridades legislativas y sanitarias tendrían que invertir en esta tecnología para garantizar su disponibilidad cuando no es factible realizar un trasplante de células madre hematopoyéticas.

Más allá de políticas nacionales deben implementarse mecanismos de colaboración internacional entre centros de referencia establecidos y países que carecen de los requerimientos financieros o técnicos para proporcionar terapias de IgG, trasplante de células madre hematopoyéticas, TG, u otros tratamientos salvadores.

De manera adicional, las autoridades de salud en cada país deberían promover el acceso a diagnósticos tempranos y a una atención especializada de calidad, para asegurar un tratamiento apropiado para todos los pacientes. Los programas de concienciación han resultado en más pacientes con inmunodeficiencias primarias diagnosticados cada año, con lo que la infraestructura existente está siendo saturada, y muchos pacientes deben viajar distancias largas para recibir atención médica adecuada. Deberían crearse, además, nuevos centros regionales especializados que permitan un acceso geográfico equitativo a personal médico y de enfermería experto en estas enfermedades; debe adiestrarse a más médicos para que puedan diagnosticar y dar atención médica adecuada al número creciente de pacientes con inmunodeficiencias primarias. Al mismo tiempo, para los pacientes pediátricos que alcanzan la edad adulta deben establecerse rutas coordinadas de transición hacia servicios de calidad para adultos que aseguren una atención planificada. 
Debido a su papel en proporcionar información de utilidad para apoyar a los médicos en la toma de decisiones, y permitir que las compañías farmacéuticas garanticen el abasto de productos médicos relevantes para cubrir la demanda, deben establecerse registros de pacientes que ayuden a evaluar la prevalencia e incidencia de las inmunodeficiencias primarias. También deben implementarse registros internacionales de pacientes que proporcionen información a los centros de países en desarrollo que no tienen redes nacionales propias.

Por último, si bien no menos importante, necesitamos promover la formación de redes profesionales que utilicen los recursos de centros especializados certificados y registros de pacientes; que establezcan lineamientos de tratamiento y eleven los estándares de la atención, y que desarrollen grupos organizados de comunicación en línea para discutir casos clínicos difíciles, y así apoyar a médicos que viven lejos de los centros especializados. ${ }^{4}$

Con diagnóstico oportuno, atención adecuada y tratamientos óptimos, las inmunodeficiencias primarias son susceptibles de tratamiento y es posible evitar discapacidades permanentes, vacunaciones innecesarias y hospitalizaciones prolongadas. Por ello, es vital que se tomen pasos decisivos en los ámbitos local, regional e internacional, para garantizar que los mecanismos mencionados sean puestos en marcha, y permitir que todos los pacientes con inmunodeficiencias primarias tengan acceso a una atención óptima a la que tienen derecho.

Varios estudios han tratado de establecer una prevalencia de inmunodeficiencias primarias en varios países, y han generado resultados inconsistentes: se estimó una frecuencia de 0.38/100,000 habitantes en México 2007, 1.94/100,000 en
Reino Unido 2011, 3.3/100,000 en Argentina 2007, 5.38/100,000 en Francia 2011, y 5.6/100,000 en Australia 2007. Con base en datos de registros, estos estimados parecen mucho menores que algunos otros fundamentados en encuestas de poblaciones específicas recientemente recabados en Estados Unidos, incluyendo la encuesta telefónica llevada a cabo por Boyle \& Buckley que reportó una prevalencia de $86.3 / 100,000$ habitantes. ${ }^{5-7}$

\section{Agradecimientos}

Saúl Lugo Reyes tradujo el original al español.

\section{REFERENCIAS}

1. WHO model list of essential medicines for children. Fifth edition. WHO; 2015. [Cited 2015 Nov 20]. Available from http://www.who.int/medicines/publications/essentialmedicines/EMLc_2015_FINAL_amended_AUG2015.pdf?ua=1

2. Clément $M C$, Mahlaoui N, Mignot $C$, Le Bihan $C$, Rabetrano H, Hoang L, Neven B, Moshous D, Cavazzana M, Blanche S, Fischer A, Audrain M, Durand-Zaleski I. Systematic neonatal screening for severe combined immunodeficiency and severe T-cell lymphopenia: Analysis of cost-effectiveness based on French real field data. J Allergy Clin Immunol. 2015;135:1589-93.

3. Ghosh S, Thrasher AJ, Gaspar HB. Gene therapy for monogenic disorders of the bone marrow. Br J Haematol. 2015 Jun 5. doi: 10.1111/bjh.13520 [Epub ahead of print]

4. Principles of care for primary immunodeficiencies. Font Immunol. [on line] 2014 Dec 14. [Cited 2015 Nov 20] Available from http://journal.frontiersin.org/article/10.3389/ fimmu.2014.00627/full

5. Bousfiha AA, Jeddane L, Ailal F, Benhsaien I, Mahlaoui N, Casanova JL, Abel L. Primary immunodeficiency diseases worldwide: more common than generally thought. J Clin Immunol. 2013;33(1):1-7.

6. Errante PR, Franco JL, Espinosa-Rosales FJ, Sorensen R, Condino-Neto A. Advances in primary immunodeficiency diseases in Latin America: epidemiology, research, and perspectives. Ann N Y Acad Sci. 2012;1250:62-72.

7. Boyle JM, Buckley RH. Population prevalence of diagnosed primary immunodeficiency diseases in the United States. J Clin Immunol. 2007;27(5):497-502. 